\section{LA-7948-MS}

Informal Report

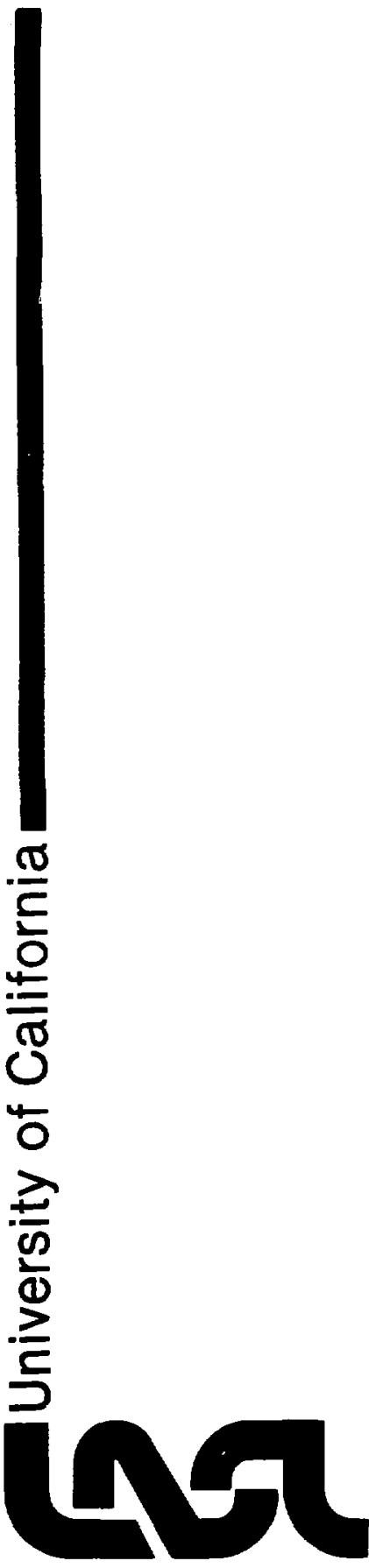

Valence Instabilities as a Possible Source of Actinide System Inconsistencies 
LA-7948-MS

Informal Report

UC-4

Issued: August 1979

\title{
Valence Instabilities as a Possible Source of Actinide System Inconsistencies
}

\author{
Thomas A. Sandenaw
}




\title{
VALENCE INSTABILITIES AS A POSSIBLE SOURCE OF
}

\section{ACTINIDE SYSTEM INCONSISTENCIES}

\author{
by
}

Thomas $A$. Sandenaw

\begin{abstract}
The presence of a mixed-valence state in light actinides appears evident from the crystal structures of certain $U, N p$, and $\mathrm{Pu}$ phases. As supporting evidence, the physical property response of these actinide elements (and some of their alloys) is compared with that of rare-earth metallic compounds known to have an unstable valence.

Impurities may stabilize an intermediate (different) valence state locally in rare-earth compounds in the presence of the valence state of the bulk phase. Impurity elements from different periodic table groupings may likewise stabilize different intermediate valence states in light actinide elements, thus contributing to inconsistencies in results reported by different experimentalists.

Any model (theory) advanced for explaining the physical property behavior of $\mathrm{U}, \mathrm{Np}$, and $\mathrm{Pu}$ may also require consideration of a configurational limit. A phenomenological connection could exist between a martensitic transformation and the fluctuation temperature in both rare earth and actinide systems.
\end{abstract}

\section{INTRODUCTION}

The results of low-temperature physical property measurements made on the early actinide elements ( $U, \mathrm{~Np}$, and $\mathrm{Pu}$ ) show a variability or inconsistency between test specimens. Results appear to have a greater dependence on impurity content than one would expect and may be a clue to the source of inconsistencies. Many theorles have been advanced for explaining the low-temperature physical property behavior of the early actinides. Most of these were proposed as explanations for the maximum in the electrical resistivity curve of $\alpha$-phase plutonium. Brief surveys of theories for $\alpha-P u$ have been given by Brodsky et al. $(1974)^{1}$, Sandenaw and Harbur $(1974)^{2}$ and Jullien and Coqblin (1975). ${ }^{3}$ Each group of these authors has stressed proposals that it considers to be of greatest importance. None of these surveys presents all of the major theories and the shortcomings of each. 
A criticism of mos: of the actinide-oriented theories presented to date is that, although they can expla1n a maximurn in resistivity curves, they cannot account for the absence of magnetic order in so many actinide materials. Jullien and Coqblin ${ }^{4}$ have proposed that the absence of localized magnetism in $\mathrm{U}, \mathrm{Np}$ and Pu metals could be accounted for by a variable d-f hybridization. There is considered to be hybridization of $f$ and $d$ states in rare-earth elements or compounds which show properties of the mixed-valence state. 5

The currently accepted crystal structures of a few phases of $U$, Np and $\mathrm{Pu}$ metals also suggest the presence of a mixed-valence state. Models presented to explain the mixed-valence state in rare-earth elements and compounds are based on valence instabilities or valence fluctuations. These models (or theories) can explain a maximum in the $\rho$ vs $T$ curves as well as a sinultaneous absence of magnetism.

Valence instabilities (fluctuations) appear to be a phenomenon that can help explain most of the observed physlcal property behavior of $\alpha$-Pu and st111 be applicable to the properties of certain plutonium alloys as well as to other light actinide eleinents and their alloys. The concept of valence instabilities has been considered by Brodsky ${ }^{6}$, but he suggested that ic must be approached cautiously in the light actinides. Valence instabilities have also been designated as interconfiguration fluctuations (ICF). An objection, that can be rais $t$ to a valence fluctuation theory for light actinides, is the absence of an appr iable lattice contraction indicative of a valence transition at a critical point. This criticism may not be valid.

There is another complicating factory. The $\beta \rightarrow \alpha$ phase transformations in $U, N p$ and $\mathrm{Pu}$ may be martensitic, as also may be their higher temperature phase transformations. ${ }^{7}$ If the $\beta$ phase is retained due to impurities or to cooling rate, then there can be low-temperature martensitic $\beta \rightarrow \alpha$ transformations in the early actinides. The case of martensitic $\beta \rightarrow \alpha$ transformations in $P u$ at room temperature and below has been discussed by White ${ }^{8}$ and that of martensitic $\delta \rightarrow \alpha$ transformations by Goldberg et al. ${ }^{3}$ The case for a martensitic transformation in $\alpha-U$ below ambient temperature has been summarized by this author ${ }^{10}$. The $\gamma \rightarrow \alpha$ transformation in cerium metal is martensitic ${ }^{11}$. A theory based on configuration crossover has been presented by Hirst $^{12}$ to explain the $\gamma \rightarrow \alpha$ phase transition in Ce metal, i.e., he considers this metal to be showing ICF behavior. This leads to the speculation that there is some connection between the mixedvalence state and a tendency for a martensite-like behavior in rare earth and actinide systems.

\section{INTERCONFIGURA'TION FLUCTUATION (ICF) THEORY}

Interconfiguration fluctuations (valence fluctuations) have been observed mainly in metallic rare-earth compounds under application of pressure or upon cooling to low temperatures. These compounds are also referred to as mixedvalence compounds because the atom-like $f$ levels and the wide $s-d$ band coexist at the Fermi level ${ }^{13}$. They are metallic in the sense that, although the $D$. C. conductivity is poor, it is still comparable to that of some metals. In the rare-earth materials showing valence instabilities, there does not appear to be either magnetic order or a temperature-dependent susceptibility. The absence of magnetism is considered to arise because of fluctuations between two ionic configurations of different valencites. 
Amolel for explaining the different integral occupations of the $4 \mathrm{f}$ shell wis first developed by llirst". Interconfiguration fluctuations are understood by llitst as arisims when the energy levels are arranged in such a way that two configurations (or valencies) compule for stability. He considers that valence fluctuations arise in a systen at configurational crossover. By this he supposedly means that the system has a many-body ground state in which each ion has an appreciable probability of being found in either of two competing configurations. The enersy neessary to take an ion from one configuration to the other is approsimately zero. The configurational change is supposedly due to the transfer of an electron to the condution band at the Fermi energy. Interconfiguration fluctuation (LCF) suggests a persistent motion of the system. Hirst ${ }^{5}$ explains that one can think of ICF as implying a quantum-mechanical zero-point moison which is implicit in the many-body ground state.

Ghatak and kvignon ${ }^{5}$ considered a theoretical model for a possible configuration crossover in rixed-valence compounds which was essentially based on a coulomb interaction between local ized and itinerant states. They incorporated hybridization between atom-1ike $F-s t a t e s$ and d-states in their model for explaining mixed-vilence electronic and elastic properties.

Tournier and Holtzberg ${ }^{16}$ surveyed the behavior of race-earth systems with an unstable valonte. They concluded that, in principle, compounds of rare carth elements with an unstable valcnce ma contain either integral valence states, a "uniform" intermediate state, or some combination. They also concluded that impurities or defects may bave a very iarge effect when two configurations are nearly degenerate. A varicty of behavior would be possible in these systems, depending upon the energy difference between configurations and the concentration and type of impurity or defect.

A tro-band picture was developed by Sales and Viswanathan ${ }^{17 a}$ for calculating the contribution of the $4[$ shell to magnetic susceptibility, specific heat, and electrical resistivity. 'The bandwidtlss in their modej are related to the \%ecman level lifetime ( $\mathrm{T}=0$ ) of each configuration via the uncertainty relation, $i_{n}, \pi / \tau_{n}, i_{n-1} \cdot \pi / r_{n-1}$. They noted that the resistivities of ICF compounds fall into two broad categories. In one the conductivity is dominated by electrons which flurtuate in and out of the $f$ shell. In the other both an "s-like" and an "f-like" band take part in the electrical conduction.

Sales ${ }^{17}$ ) notes that it is experimentally difficult to distinguish between a rare-earth JCF and Kondo sy..tem. He suggests that there is a single physical mechanism responsible for the entire spectrum of magnetic behavior. That mechanism should iscorporate the Kondo, spin fluctuation, and valence fluctuation models as liniting cases.

\section{T.II. APPLICABIJITY OF ICF THEORY TO ACTINIDE SYSTEMS}

Preeman and koelling ${ }^{18}$ have noted that the $5 f$ electrons in the actinides are not well localized in the first part of the series. Their itinerant nature makes them hybridize strongly with the $6 \mathrm{~d}$ and $7 \mathrm{~s}$ bands. Hybridization is considered in a model used to explain properties of rare-earth ICF compounds ${ }^{5}$. A non-integer occupation of the $5 f$ shell may be a consequence of hybridization. Ward and $H i 11^{19}$ have stated that hybridized bonding states of the early actinide metals, $\mathrm{Pa}, \mathrm{U}, \mathrm{Np}, \mathrm{Pu}$ and possibly $\mathrm{Am}$, do not admit to a definable valence. It should be appreciated that in the discussion of metallic rare-earth compounds, the hybridization is considered to be among ionic states. 
The crystal structures of certain $U$, Np and $P u$ metal phases suggest the presence of the mixed-valence state. There are 30 atoms per unit cell in the tetragonal structure of $\beta$-phase $U$. ntoms appear to be present in the $\beta-U$ structure in four different electronic states with valencies of 3,4 , 5 , and 6 respectively, as pointed out by Thewlis and Steeple ${ }^{20}$. Fournier ${ }^{2}$ nas also pointed out that there are inequivalent sites in the crystal structures of $\alpha-\mathrm{Np}$ and $\alpha-\mathrm{Pu}$. He notes that the inequivalent sites correspond to different configurations with the inequivalent sites having about the same stability in both neptunium and plutonium. The existence of different states for different $\alpha$-Np atoms appears to have already been provided by the experimental evidence of Dunlap et $a 1.2$. Eight inequivalent atom sites exist in the primitive monoclinic unit cell of $\alpha$-phase $P u$ and seven different atomic sites exist in the b.c. monoclinic unit cell of $\beta-P u$. Long ${ }^{2}$ has commented that in the similar but not identical case of $\alpha-M n$, the different atomic sites carry different magnetic moments, which means a different electronic correlation on each type of site.

The known physical property response of $\alpha-\mathrm{Pu}$ and other actinide metals and alloys makes an explanation based on interconfiguration fluctuations (ICF) very attractive. $\Lambda$ discussion of similarities between the physical properties of the actinide materials and ICF compounds is presented in the following section.

\section{SIMILARITIES IN THE PHYSICAL PROPERTY RFSPONSES OF ACTINIDE MATERIALS AND ICF COMPOUNDS}

There are similarities between the physical property behavior of actinide materials and ICF compounds. These similarities are as follows: a maximum in electrical resistivity curves, an absence of magnetism, an unusually high electronic specific heat, a change in lattice constant upon cooling or upon application of pressure, a humping in thermoelectric power versus temperature curves, a minimum in Young's modulus curves, and an extreme sensitivity to impurities.

Alpha-phase $\mathrm{Pu}, \mathrm{Pu}_{6} \mathrm{Fe}, \mathrm{PuZn_{2 }}, \mathrm{PuA1}{ }_{2}$ and $B-\mathrm{Pu}$ have widely differing crystal structures as noted by Brodsky et al. ', yet a maximum in $\rho$ vs $T$ curvis has been observed for all of these materials. It seemed improbable to these authors that the listed plutonium phases and alloys all had similar $f$ bands and appropriate values of $\mathrm{N}^{\prime}(\mathrm{E})$ and $\mathrm{N}^{\prime \prime}(\mathrm{E})$. According to Sales and Viswanathan ${ }^{17 a}$, the resistivities of ICF compounds fall phenomenologically into two broad categories. In one of these the conductivity appears to be dominated by the very electrons which fluctuate in and out of the $f$ shell. The effect is large and the resistivities of these compounds increase with decreasing temperature. Interconfiguration fluctuation compounds showing this behavior are $\mathrm{CeCu}_{2} \mathrm{Si}_{2}{ }^{17 \mathrm{a}}$,

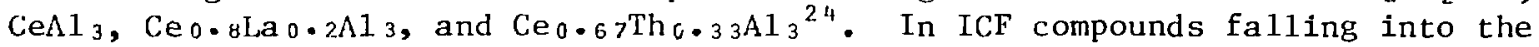
second category, both an "s-like" and an "f-like" band take part in the conduction to yield an S-shaped resistivity curve ${ }^{17}$. Other actinide materials appear to show a resistivity behavior which falls into this second category ${ }^{1}$.

Alpha-phase plutonium and many other actinide materials show a large paramagnetic, but temperature-independent magnetic susceptibility with no evidence for magnetic order. Recent experimental evidence indicates an apparent loss of ionic magnetic moment in several metallic rare-earth systems 17,25 . This demagnetization has been interpreted as a temporal fluctuation between two ionic-like configurations ${ }^{17 a}$.

Hirst ${ }^{15}$ expects a large electronic specific-heat coefficient for an ICF system, one with a coefficient orders of magnitude larger than for normal metals. 
Iarge values have been reported for the electronic contribution to specific heat of $x-\mathrm{Pu}^{2}, 25$ and for other actinide materials ${ }^{5}$. This behavior has not only been

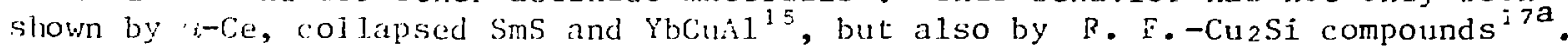
An appreciable lattice contraction is usually observed at the critical point for a valence transition in an ICF compound. There appears to be a density maximum in both $t_{-1} \mathrm{U}^{27}$ and $\mathrm{is}_{-} \mathrm{P}^{28}$ at about $50 \mathrm{~K}$. The lattice change is not great in these two actinide elements, but it could indicate a critical point. An appreciable lattice contraction may not always occur with a valence transition. It should be noted that each atom in a-phase $U$ is considered to be equivalent. This phase is not mixed valent at room temperature.

There is a similarity between the thermoelectric power vs temperature hehavior of liglit actinide elements and ICF compounds. A large maximum has been seen in the thermoelectric power curves of R. F. $-\mathrm{Cu}_{2} \mathrm{Si}_{2}$. compounds ${ }^{17 \mathrm{a}}$. The TEP vs $T$ curve for $\left(x-\mathrm{Np}^{29}\right.$ is broad and very similar to that curve shown by $\mathrm{EuCu}_{2} \mathrm{Si}_{2}{ }^{17 \mathrm{a}}$. Humping has been observed in the low-temperature thermoelectric power curves of $x-U^{30}$ and $i-P u^{28}, 29$ with peaking appearing, respectively, at $33-40 \mathrm{~K}$ and $60 \mathrm{~K}$. Sales and Viswanatian 7 a have noted that peaking in thermoelectric power occurs near the rluctuation temperature.

Elastic measurements made across the valence transition of two Ce $(1-x)^{\text {Th }} x$ samples have shown a softening of the Young's modulus at the transition $^{3 !}$. A slight dip was Iikewise seen by Lallement ${ }^{2}$ in the Young's modulus vs temperacure curve of $x-\mathrm{Pu}$, with the minimum appearing at $\leadsto 60 \mathrm{~K}$. A similar curve for $a-U$, as letermined by Rosen ${ }^{33}$, sliowed a minimum at $235 \mathrm{~K}$.

As noted above, Tournier and Hol tzberg ${ }^{16}$ concluded that impurities or defects could have a considerable jnfluence on properties of rare-earth materials with an unstable valence. The concentration of impurities in $\alpha$-Pu, in particular, appears to have an unusual influence on low-temperature properties. Joel et: al. ${ }^{34}$ showed that small amounts of certain impurities had a large effect on the $\rho$ vs T curves.

The recently reported low-temperature $\rho$ vs $T$ and $C_{p}$ vs $T$ behavior of ${ }^{24}{ }^{1} \mathrm{Am}$ and ${ }^{21.3} \Lambda \mathrm{m}^{35}$ suggests that americium may also be showing valence instabilities. This is because of slightly higher resistivity values between $50-60 \mathrm{~K}$ along with heat capacity peaking between $\approx 50-70 \mathrm{~K}$. The magnetic susceptibility of ${ }^{241} \mathrm{Am}$ is almost temperature independent down to $100 \mathrm{~K}$ and there is no indication of magnetic ordering below this temperature ${ }^{36}$. The fluctuation temperature for Am may be very near to those temperatures speculated above for $\alpha-U$ and $y-P u$, because of a similarity in heat capacity peaking.

\section{DISCUSSION}

The crystal structures of certain $\mathrm{U}$, Np, and $\mathrm{Pu}$ phases suggest the presence of a mixed-valence state. A comparison of the physical property response of interconfiguration fluctuation (ICF) compounds and the early actinides (and some of their alloys or compounds) indicates that a theory based on ICF models could be applicable to these actinicles. Hirst ${ }^{14}$ has pointed out the advantage of considering the problem of ? magnetic ion in a metal from the viewpoint of an ionic or configuration limit rather than from a limit of largely delocalized electrons.

The best support for considering ICF models for actinides is the cemperature-independent magnetic susceptibility and lack of magnetic order in so many actinide materials. The susceptibility results must reflect an average value for the different ionic configurations present in the actinide elements, with intrinsic moment fluctuations preventing magnetic ordering. The observed 
phenomenon that the resistivities of ICF R. E.-compounds can fall into two broad categories also gives support for considering ICF models for $U$, Np, arid $\mathrm{Pu}$, and perhaps even $\mathrm{Am}$.

The effects of impurities can be significant upon elements capable of existing in the mixed-valence state. The variability of reported results for actinide metals and their alloys may be explained as basically an impurity effect. Physical properties depending upon electron states, e.g., thermoelectric power, electronic contribution to heat capacity, and electrical conductivity, could show slightly different values at a particular temperature in the presence of different impurities. The best support for this speculation is given by the resistivity study of $\alpha$-phase $P u$ made by Joel et al. ${ }^{34}$. Their study showed the effect of different impurity groups upon not only residual resistivity, $\rho_{\max }$, and resistivity at $300 \mathrm{~K}$, but also upon specimen density. It is suggested that elements from different columns of the periodic table are able to contribute or transfer a different number of electrons to the conduction band of $\alpha-\mathrm{Pu}$ atoms. The configurational change may be different in light actinide elements, depending upon the number of electrons transferred by the impurity elements present to the conduction band at the Fermi energy.

The $\gamma \rightarrow \alpha$ phase transformation in Ce metal has been associated with a configuration crossover ${ }^{12}$. This transformation is considered to be martensitic. Several phase transformations in light actinide elements have been shown to be either martensitic or diffusion controlled. This writer has presented evidence for the possibility of a martensitic transformation in $\alpha$-phase $U$ in the range $36-50 \mathrm{~K}^{10}$. It was noted above that the fluctuation temperature for $\alpha-U$ nay be within this same temperature range. One can therefore speculate that there may be a phenomenological connection between the fluctuation temperature of the mixed-valence state and certain martensitic transformations in rare-earth and actinide systems.

The present theory for the behavior of intermediate valence compounds is still considered to be incomplete. An exact theory for explaining light actinide behavior needs to be developed. It may turn out that the final model (theory) will explain the Kondo effect and spin and valence fluctuations in both rareearth and actinide systems.

\section{ACKNOWLEDGEMENTS}

I want to thank J. A. O'Rourke, $\mathrm{CMB}-8$, for helpful comments and suggestions during preparation of this paper.

\section{REFERENCES}

1. M. B. Brodsky, A. J. Arko, A. R. Harvey and W. J. Nellis, "Transport Properties," The Actinides, Vol. II, A. J. Freeman and J. B. Darby, Jr. Eds. (Academic Press, New York, 1974) Chap. 5, pp. 185-264.

2. T. A. Sandenaw and D. R. Harbur, "Evidence for an Antiphase Type of Structure in Pu Metal and Its Alloys." J. Phys. Chem. Solids, 35, 795-305 (1974).

3. R. Jullien and B. Coqblin, "Effect of Spin Fluctuations in Actinide Systems," Pu 1975 and Other Actinides, H. Blank and R. Lindner, Eds. (North-Holland Pub1. Co., Amsterdam, 1976) pp. 425-446. 
4. R. Jullien and B. Coqblin, "Study of the d-f Hybridization in Actinide Metals and Alloys," Phys. Rev. B, 8, 5263-5271 (1973).

5. S. K. Chatak and M. Avignon, "A Theoretical Model of Mixed Valence Electronic and Elastic Properties," Valence Instablities and Related Narrow-Band Phenomena, R. D. Parks, Ed. (Plenum Press, New York, 1977) pp. 2.29-246.

6. M. B. Brodsky, "Experimental Studies of Narrow Band Effects in the Actinides," Ref. 5, pp. 351-362.

7. A. Goldberg and T. B. Massalski, "Phase Transformations in the Actinides," Plutonium 1970 and Other Actinides, W. N. Miner, Ed. Nuclear Metallurgy, Vo1. 17, Part II (AIME, New York, 1970) pp. 875-973.

8. J. S. White, "The Veining Phenomenon in Unalloyed Plutonium," J. Nucl. Mater. 59,77-85 (1976).

9. A. Goldberg, R. L. Rose and J. C. Shyne, "Effects of Stress and Plastic Deformation on the Transformation of the Delta Phase in a Pu-1 at \% Ga Alloy," J. Nucl. Mater. 55, 33-52 (1975).

10. T. A. Sandenaw, "Variable Low-Temperature Martensitic Behavior of AlphaPhase Uranium," Scripta Met. 12 , 39-41 (1978).

11. M. S. Rashid and C. J. Alstetter, "Allotropic Transformations in Cerium," Trans. AIME, 236, 1649-1656 (1966)(and references therein).

12. L. L. Hirst, "Configuration Crossover in $4 \mathrm{f}$ Substances Under Pressure," J. Phys. Chein. Solids, 35, 1285-1296 (1974).

13. C. M. Varma, "Mixed-Valence Compounds," Rev. Mod, Phys. 48, 219-238 (1976).

14. L. L. Hirst, Phys. Kondens, Mater. 11, 255 (1970).

15. L. L. Hirst, "Electronic Properties of $4 \mathrm{f}$ Substances at Configuration Crossover, "Phys. Rev. B, 15, 1-9(1977).

16. R. Tournier and F. Holtzberg, "Observation of Localized Moments, Kondo Effect, Spin Glass Regime, Magnetic Ordering in Rare Earth System with Unstable Valence," Ref. 5, pp. 3n3-314.

17. a) B. C. Sales and R. Viswanathan, "Demagnetization Due to Interconfiguration Fluctuations in the $\mathrm{RE}-\mathrm{CL}_{2} \mathrm{Si}_{2}$ Compounds," J. Low Temp. Physics, 23, 449-467 (1976). b) B. C. Sales, "A Model for the Thermodynamic Properties of Metallic Rare Earth Systems with an Unstable Valence," J. Low Temp. Physics, 28, 107-127 (1977).

18. A. J. Freeman and D. D. Koeliing, "Electronic Energy Band Structure of the Actinide Metals," Ref. 1, Vol. I, Chap. 2, pp. 51-108.

19. J. W. Ward and H. H. Hill, "An Entropy Correlation for the $4 \mathrm{f}$ and $5 f$ Metals: Relation of Electronic Properties to Metallic Radii, Magnetic Transformations and Thermodynamics of Vaporization," Heavy Element 
Properties, W. Müller and H. Blank, Eds. (North-Holland, Amsterdam, 1976) pp. 65-78.

20. J. Thewlis and H. Steeple, "The B-Uranium Structure," Acta Cryst. Z, 323328 (1954).

21. J.-M. Fournier, "Bonding and the Electronic Structure of the Actinide Metals," J. Phys. Chem. Solids, 37, 235-244 (1976).

22. B. D. Dunlap, M. B. Brodsky, G. K. Shenoy and G. M. Kalvius, "Hyperfine Interactions and Anisotropic Lattic Vibrations of $23{ }^{37} \mathrm{~Np}$ in $\alpha$-Np Metal," Phys. Rev. B, 1 , 44-49 (1970).

23. K. A. Long, "The Role of Lattice Vibrations in the Determination of the Physical Properites of the Actinides," Ref. 3, p. 311-322.

24. K. H. J. Busciow and H. J. van Daal, "Investigations on the Resistivity of the Compound $\mathrm{CeAl}_{3}$," Solid State Comm. $\underline{8}$, 363-365 (1970).

25. B. C. Sales and D. K. Wohlleben, "Susceptibility of InterconfigurationFluctuation Compounds," Phys. Rev. Letters, 35, 1240-1244 (1975).

26. T. A. Sandenaw, "Difficulties in Producing Pure $\alpha$-Phase Plutonium," J. Nuc1. Mater. 73, 204-207 (1978).

27. A. F. Schuch and H. L. Laquer, "Low Temperature Thermal Expansion of Uranium," Phys. Rev. $\underline{86}, 803$ (1952).

28. R. Lallement, "Dilatation Et Pouvoir Thermoelectrique Du Plutonium $\alpha$ A Basse Temperature," J. Phys. Chem. Solids, 24, 1617-1624 (1963).

29. G. T. Meaden and J. A. Lee, "The Thermoelectric Powers of the Actinide Metals at Low Temperatures," Cryogenics, 2, 182 (1962).

30. N. H. Sze and G. T. Meaden, "Study of the Occurrance of Different a-Phases of Uranium at Low Temperatures by Thermopower and Resistivity Experiments," Ref. 7, pp. 974-982.

31. M. C. Croft and R. D. Parks, "Elastic Measurements Near a Valence Transition," Ref. 5, pp. 455-458.

32. R. Lallement, "Mesure Du Module D'Young Du Plutonium Alpha $A$ Basse Temperature," Phys. Letters, 5, 182-183(1963).

33. M. Rosen,"Elastic Moduli and Ultrasonic Attenuation of Polycrystalline Uranium from 4.2 to $300^{\circ} \mathrm{K}$," Phys. Letters, 28A, 438-439 (1968).

34. J. Joe1, J. Jodet and F. Wandet, "Effet Des Impuretes Sur La Resistivite Electrique Du Plutonium En Phase Alpha A Basses Temperatures: Correlation Entre La Purete Chimique F.t La Resistivite Residuelle - Cas Particulier De L'Americium," J. Nuc1. Mater. 60, 203-215 (1976). 
35. W. Müller, R. Schenkel, H. E. Schmidt, J. C. Spirlet, D. L. McE1roy R. O. A. Hall and M. J. Mortimer," "The Electrical Resistivity and Specific Heat of Americium Metal," J. Low Temp. Phys. 30, 561-578(1978).

36. B. Kanellakopulos, A. Blaise, J. M. Fournier and W. Müller, "The Magnetic Susceptibility of Americium and Curium Metal, "Solid State Comra.17, 713-715(1975). 